\title{
«Goldstandard» als Innovationskiller?
}

Adrian Merlo

\section{Die Innovation war nicht gegeben, weil zwei Jahre zuvor eine Studie mit leicht besseren mittleren Überlebenszahlen veröffentlicht worden war}

Tempora mutantur, et nos mutamur in illis. Eine Ärztegeneration später wurde im gleichen Medizinjournal, dessen Ruf durch kritische Veröffentlichungen zweier langjähriger Chefredaktoren zwischenzeitlich etwas ramponiert worden war [2, 3], 2005 eine weitere Glioblastomstudie publiziert [4]. Fortschritte in Bildgebung, mikrochirurgischer und anästhesiologischer Technik, der Einsatz der Chemotherapie und eine bessere Selektion der Fälle hatten 25 Jahre nach der Walker-Studie zu einer diskreten Verbesserung des mittleren Überlebens geführt, nämlich jetzt von 12,1 Monaten mit Chirurgie und alleiniger Radiotherapie zu 14,6 Monaten mit zusätzlicher Chemotherapie. Auch dies war ein bescheidenes Resultat, insbesondere in Anbetracht des viel grösseren Aufwandes.

In dieser Nachfolgestudie wurde Temozolamid (TMZ) als alkylierendes Zytostatikum eingesetzt, das Jahrzehnte früher entdeckt worden war. TMZ kam nun aber nicht erst nach Abschluss der externen Strahlentherapie, sondern schon während der Radiotherapie in kontinuierlicher niedrigerer Dosis zum Einsatz, in der Vorstellung, dass es sich um eine strahlensensibilisierende Substanz handeln könnte. Diese grossangelegte EORTC-NCIC-Studie blieb jedoch die Antwort schuldig, ob dieses Regime tatsächlich die Empfind-

Korrespondenz: Prof. Dr. Adrian Merlo Klinik Sonnenhof Bucherstrasse 30 CH-3006 Bern

adrian.merlo@gmx.ch lichkeit auf die Strahlentherapie erhöht, weil der entsprechende Kontrollarm fehlte, der diese Frage beantwortet hätte. Immerhin, experimentelle Daten und die vermehrte Beobachtung von symptomatischen Radionekrosen im Radiochemotherapiearm können als Hin- weis auf eine mögliche Strahlensensibilisierung durch TMZ gelten.

Erstaunlich an dieser EORTC-NCIC-Studie aber war, dass sie überhaupt im NEJM zur Publikation angenommen wurde, war doch die Innovation nicht gegeben, weil zwei Jahre zuvor eine sehr ähnliche Studie der Deutschen Arbeitsgemeinschaft für Neuro-Onkologie (NOA) veröffentlicht worden war, die zu sogar leicht besseren mittleren Überlebenszahlen von 17,3 Monaten geführt hatte [5]. Merkwürdigerweise war diese im Jounal of Clinical Oncology publizierte Arbeit in der NEJM-Publikation nicht zitiert worden, was schwerverständlich ist, da einige Autoren an beiden Studien teilgenommen hatten. Während also die NOA-Studie ignoriert wurde, hat die NEJM-Studie einen schwernachvollziehbaren therapeutischen Enthusiasmus ausgelöst, ja es war sogar von einem «Durchbruch» in der Behandlung des Glioblastoms die Rede.

Ob die unterschiedliche Rezeption der beiden Studien direkt proportional zur Höhe des Werbebudgets im weitesten Sinne war, wäre auf jeden Fall zu untersuchen, wenn man den beiden ehemaligen NEJMChefredaktoren Angel und Kassirer Glauben schenken will, die sich pointiert gegen die zu starke Einflussnahme der Pharmaindustrie auf die klinische Medizin geäussert hatten [2, 3]. Wenigstens in der Tagespresse (NZZ 10.3.2005 und 23.3.2005) gab es einige wenige kritische Kommentare, doch kaum in der Fachliteratur, mit Ausnahme etwa der im Cancer publizierten Bemerkungen einer deutschen Forscherin, die von den Herausgebern des NEJM und auch des Lancet Oncology, wo 2008 das 5-Jahres-Studien-update veröffentlicht worden war [6], zurückgewiesen wurden. Freundlicherweise haben die Herausgeber des anerkannten amerikanischen Fachjournals Cancer die beiden lesenswerten Kommentare dennoch publiziert [7, 8]. In ihrem ersten Kommentar argumentiert die Autorin aus dem Forschungszentrum Jülich ökonomisch, indem sie die Frage aufwirft, ob die zehnfache Preisdifferenz zwischen TMZ und dem altgedienten Zytostatikum ACNU (€ 20000.- versus € 2000.- pro Fall) bei vergleichbarer Wirksamkeit in einer palliativen Konstellation gerechtfertigt sei [7]. Der Kommentar wurde von der angesteuerten Ärzteschaft ignoriert.

Gegen einen hohen Preis für ein echt innovatives Medikament ist grundsätzlich nichts einzuwenden, im Gegenteil, aussergewöhnliche wissenschaftliche und pharmazeutische Leistungen müssen belohnt werden. Geradezu gebetsmühlenartig wurde aber nun der sog. «Durchbruch» in der Behandlung des Glioblastoms rezitiert, auch an zu kritischem Denken verpflichteten Universitätskliniken, und sehr rasch war von einem neuen «Goldstandard» die Rede, der zwingend anzuwenden sei, ja dessen Nichtanwendung so- 
gar unethisch sei. In ihrem zweiten, kürzlich ebenfalls im Cancer erschienenen Kommentar als Reaktion auf das Studien-update [6] wählte die Forscherin aus Jülich deutlich kritischere Worte, die grundsätzliche Fragen über die Integrität der 2005 erschienenen NEJM-Studie aufwerfen [4], da z. B. der wiederholte Hinweis auf die Kontamination der Daten mit prognostisch günstigeren Hirntumoren als das Glioblastom nicht zu Korrekturen oder zumindest zur Offenlegung der Daten geführt hat, womit die Zahlen über sog. Langzeitüberlebende uninterpretierbar werden.

Der Weg zu einer wirksameren Behandlung des Glioblastoms fängt mit der kritischen Analyse des status praesens an. Der lockere Umgang mit Begriffen wie «Durchbruch» und «Goldstandard» - «Gold» bezieht sich ja kaum auf den Jahresumsatz eines Medikamentes - droht den Begriff des medizinischen Fortschritts zu verwässern, ja in diesem Bereich ad absurdum zu führen. Translationelle klinische Forschung bedeutet unter anderem die Entwicklung neuartiger Therapieformen. «Neu» kann doch nicht Neuverpackung eines schon hinlänglich bekannten Therapiekonzeptes bedeuten.

Die universitäre und pharmazeutische Forschung hat in den letzten Jahren vielversprechende Zielmoleküle definiert, die zu testen sind [9]. Vorstellbar sind beispielsweise kombinatorische Ansätze mit jeweils begrenzten Patientenzahlen pro Subgruppe, wobei innerhalb des gleichen oder zwischen zwei unterschiedlichen Signalübermittlungspfaden Synergien gesucht werden. Dieser Ansatz zielt auf die Entdeckung eines stochastischen Glückswurfs ab in Form eines starken therapeutischen Effekts. Solche klinische Versuche könnten sogar präoperativ durchgeführt werden, was die Untersuchung des anschliessend entfernten Tumorgewebes auf Behandlungseffekte erlaubte. Neben solch aufwendigen klinisch-experimentellen Studien gilt es, auch neue Medikamente in die Standardbehandlung einzubeziehen resp. die momentane Standardbehandlung zu verbessern. Bei der externen Strahlentherapie besteht vermutlich noch Verbesserungspotential, wenn es gelingt, die Strahlenfelder besser der neurochirurgisch-neuroradiologischen Navigation und Funktionszuordnung mit Diffusion Tensor Imaging und funktionellem MRI anzupassen. Dies dürfte eine Dosissteigerung bei verminderter Toxizität des angrenzenden Normalhirns gestatten, evtl. sogar unter Verkürzung der Therapiedauer, was die Lebensqualität verbessern dürfte. Das hinter der Bluthirnschranke liegende cerebrale Interstitium, der chaotisch strukturierte Extrazellulärraum des Tumors, ist als therapeutisches Kompartiment für lokale Applikationsformen ebenfalls noch wenig erforscht [10].

Zusammenfassend stellt sich die Frage nach dem Sinn des Begriffs «Goldstandard» in einer sehr palliativen Konstellation. Das zu frühe Ausrufen von neuen Therapiestandards durch ärztliche Opinion Leaders im Verbund mit Pharma-Hochglanzbroschüren - weitere Beispiele sind Iressa für Lungenkarzinome (NSCLC) oder die Avastinerhaltungstherapie bei metastasierendem Colonkarzinom - sollte unbedingt vermie-

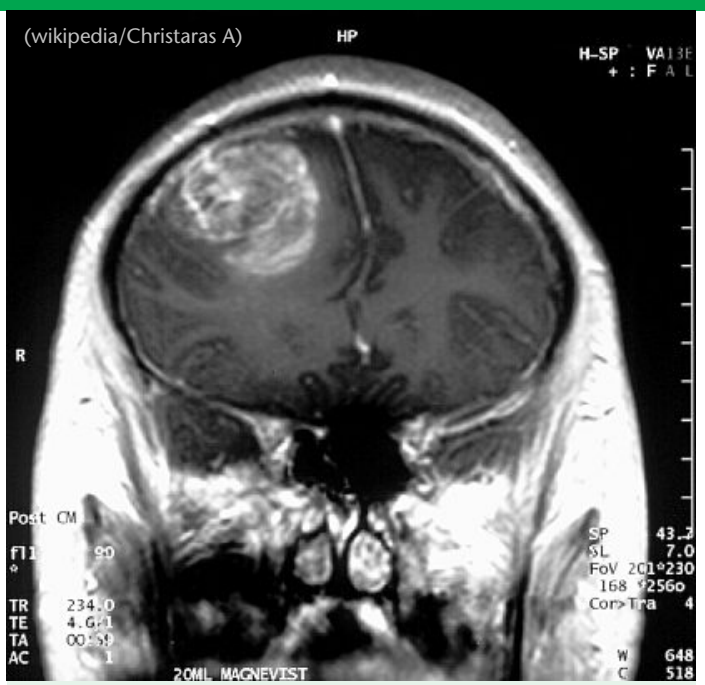

Bei einem 15-jährigen Buben zeigt das MRI ein Glioblastom (WHO-Grad IV).

den werden. Der territorial und pekuniär motivierte Gebrauch des Begriffs «Goldstandard» behindert letztlich Innovationen und damit das zukünftige therapeutische Denken und Handeln.

\section{Literatur}

1 Walker MD, Green SB, Byar DP, Alexander EJ, Batzdorf U, Brooks WH et al. Randomized comparisons of radiotherapy and nitrosoureas for the treatment of malignant glioma after surgery. N Engl J Med. 1980; 303(23):1323-9.

2 Angel M. The truth about the drug companies. How they deceive us and what to do about it. New York: Random House; 2004.

3 Kassirer JP. On the take. How medicine's complicity with big business can endanger your health. Oxford University Press; 2005.

4 Stupp R, Mason WP, van den Bent MJ, Weller M, Fisher $\mathrm{B}$, Taphoorn MJ et al. European Organisation for Research and Treatment of Cancer Brain Tumor and Radiotherapy Groups; National Cancer Institute of Canada Clinical Trials Group. Radiotherapy plus concomitant and adjuvant temozolomide for glioblastoma. N Engl J Med. 2005;(352):987-96.

5 Weller M, Muller B, Koch R, Bamberg M, Krauseneck P; Neuro-Oncology Working Group of the German Cancer Society. Neuro-Oncology Working Group 01 trial of nimustine plus teniposide versus nimustine plus cytarabine chemotherapy in addition to involved-field radiotherapy in the first-line treatment of malignant glioma. J Clin Oncol. 2003(21):3276-84.

6 Stupp R, Hegi ME, Mason WP, van den Bent MJ, Taphoorn MJ, Janzer RC et al; European Organisation for Research and Treatment of Cancer Brain Tumour and Radiation Oncology Groups; National Cancer Institute of Canada Clinical Trials Group. Effects of radiotherapy with concomitant and adjuvant temozolomide versus radiotherapy alone on survival in glioblastoma in a randomised phase III study: 5-year analysis of the EORTC-NCIC trial. Lancet Oncol. 2009;(10):459-66.

7 Linz U. Chemotherapy for glioblastoma: is costly better? Cancer. 2008;(113):2617-22.

8 Linz U. Commentary on effects of radiotherapy with concomitant and adjuvant temozolomide versus radiotherapy alone on survival in glioblastoma in a randomised phase III study: 5-Year analysis of the EORTC-NCIC trial. Lancet Oncol. 2009(10):459-66. Cancer. 2010 Feb 11 (epub ahead of print).

9 Lino M, Merlo A. Translating biology into clinic: the case of glioblastoma. Curr Opin Cell Biol. 2009(21):311-6.

10 Cordier D, Forrer F, Bruchertseifer F, Morgenstern A, Apostolidis C, Good S et al. Targeted alpha-radionuclide therapy of functionally critically located gliomas with (213)Bi-DOTA-[Thi (8), Met(O (2) (11)]-substance P: a pilot trial. Eur J Nucl Med Mol Imaging. 2010 Feb 16 [epub ahead of print]. 